\title{
Sinus Histiocytosis
}

National Cancer Institute

\section{Source}

National Cancer Institute. Sinus Histiocytosis. NCI Thesaurus. Code C85069.

A morphologic finding indicating the presence of histiocytic infiltrates within distended lymph node sinuses. 